\title{
Performance Comparison of DCT and Walsh Transforms for Watermarking using DWT-SVD
}

\author{
Dr. H. B. Kekre \\ Senior Professor \\ Computer Engineering Department \\ SVKM's NMIMS (Deemed to be \\ University), Vileparle, Mumbai,
}

\author{
Dr. Tanuja Sarode \\ Associate Professor \\ Computer Department, \\ Thadomal Shahani Engg. College, \\ Bandra, Mumbai 50, India
}

\author{
Shachi Natu \\ Assistant Professor, \\ Information Technology Department \\ Thadomal Shahani Engg. College \\ Bandra, Mumbai 50, India
}

\begin{abstract}
This paper presents a DWT-DCT-SVD based hybrid watermarking method for color images. Robustness is achieved by applying DCT to specific wavelet sub-bands and then factorizing each quadrant of frequency sub-band using singular value decomposition. Watermark is embedded in host image by modifying singular values of host image. Performance of this technique is then compared by replacing DCT by Walsh in above combination. Walsh results in computationally faster method and acceptable performance. Imperceptibility of method is tested by embedding watermark in HL2, HH2 and HH1 frequency subbands. Embedding watermark in $\mathrm{HH} 1$ proves to be more robust and imperceptible than using HL2 and HH2 sub-bands.
\end{abstract}

Keywords-Discrete Wavelet Transform (DWT); Discrete Cosine Transform (DCT); Singular Value Decomposition (SVD); watermarking.

\section{INTRODUCTION}

Advancement in technology has resulted in use of digital data which includes text, images, audio, video and multimedia data. Technology has also made it easy to duplicate/manipulate the contents of these data by various means. Piracy is a very good example of this. Thus authentication of data becomes obvious requirement before it is made available as digital data. Authentication includes information of owner of data within data itself to avoid taking undue credit as well as to prevent tampering of data. Digital watermarking is one of the most popular techniques used for digital data authentication.

Watermark is secret information which is embedded into a digital signal. Digital signal into which watermark is embedded is called as host signal or cover signal. Host signal can be text, image, audio or video data. Depending on the type of host signal, watermarking is classified as text watermarking, image watermarking, audio watermarking and video watermarking[1]. Image watermarking can be further classified into transform domain watermarking and spatial domain watermarking based on how the watermark is embedded into an image. Transform domain watermarking is one in which image is first transformed using appropriate transformation technique and then watermark is embedded into transformed coefficients of image. Spatial domain watermarking refers to directly modifying pixel values of an image to embed watermark into it. Transform domain watermarking is complex as compared to spatial domain watermarking but it is more robust also. Watermarking technique is said to be robust with respect to transformations, if watermark embedded into digital image can be easily extracted even if any attempts are made to change the data contents thereby degrading the host image. Discrete Wavelet Transform[2],[8],[13] and Discrete Cosine Transform[3],[8],[13] are most popular transforms used for Transform domain watermarking. Singular Value Decomposition[4],[8] is yet another popular approach for the same. In this paper an attempt has been made to exploit strengths of all these techniques to provide robust and imperceptible watermarking technique. Other characteristics of a good watermarking technique are perceptibility and security. Perceptibility refers to the ability to notice existence of watermark into image. Low perceptibility is desirable. Security of watermarking algorithm refers to inability to extract data contents by unauthorised party even after knowing embedding and extraction algorithm.

\section{RELATED WORK}

In literature, various approaches have been tried out for digital watermarking using wavelet transform and singular value decomposition. Xi-Ping and Qing-Sheng Zhu [5] have proposed a wavelet based method using sub-blocks of image. Instead of applying wavelet transform on whole image, it was applied to local sub-blocks. These sub-blocks were randomly extracted from original image. Watermark was embedded into part of frequency coefficients of these sub-bands by computing their statistical characteristics. A Mansouri, A Mahmoudi Aznaveh, F Torkamani Azar [6] have proposed a method using Complex Wavelet Transform (CWT) and singular value decomposition (SVD). The watermark was embedded by combining singular values of watermark in LL band of transformed image. The method proposed by them is non-blind watermarking because singular values of original image are required in extraction phase. Rashmi Agarwal and K. Venugopalan [7] have proposed a SVD based method for watermarking of color images. Each plane of color image is separately treated for embedding and extracting process. Different scaling factors were used to test the robustness of their method. Satyanarayana Murty. P. and P. Rajesh Kumar[8] have proposed a hybrid DWT-DCT-SVD based approach. HL frequency band was selected by them for embedding purpose. Method proposed in this paper is 
motivated by their work. Satendra Kumar, Ashwini Kumar Saini, Papendra Kumar[9] have also proposed a watermarking scheme based on discrete wavelet transform and singular value decomposition. They have used three level wavelet transform and then by modifying singular values of cover image, watermark is embedded into it. Medium frequency bands i.e. HL3 and LH3 were preferred for embedding. PSNR and Normalized Cross Correlation (NCC) values were used to measure the effectiveness of the method. Krishnamoorthi and Sheba Kezia[10] proposed a watermarking technique based on orthogonal polynomial based transformation for copyright protection of digital images. A visual model was used to determine strength of watermarking. This visual model was used to generate Just Noticeable Difference (JND) by analyzing low level image characteristics like texture, edges and luminance of cover image in polynomial based transformation domain. Ko-Ming-Chan and Long-Wen Chang[11] have proposed a watermarking system which embeds two different watermarks -robust and fragile into spatial and frequency domain separately. Robust watermark is embedded in wavelet coefficients of LL band whereas fragile watermark is embedded in least significant bits of watermarked image. Advanced encryption standard- Rijndeal block cipher was used to make watermarking technique public. Veysel Atlantas, A Latif Dogan, Serkan Ozturk [12] proposed a DWT-SVD based watermarking scheme using Particle Swarm Optimizer (PSO). Singular values of each subband of cover image are modified by different scaling factors. Modifications were further optimized using PSO to obtain highest possible robustness.

\section{Discrete WAVELET TRANSFORM, Discrete Cosine TRANSFORM AND SINGULAR VALUE DECOMPOSITION}

\section{A. Discrete Wavelet Transform(DWT) [13]}

Wavelets are special mathematical functions that represent scaled and shifted copies of finite length waveform. DWT is based on wavelets and analyzes the signal into its frequency components at multiple resolutions. Applying wavelet transform on two dimensional images divides image into four sub-bands LL, LH, HL and $\mathrm{HH}$ which consist of low frequency, middle frequency and high frequency components of an image. Maximum energy of an image is concentrated in LL sub-band whereas high frequency components in $\mathrm{HH}$ subband correspond to edges and textures [8]. Hence imperceptible watermarking can be achieved by using these high frequency components for embedding.

\section{B. Discrete Cosinet Transform(DCT)}

Discrete Cosine Transform converts the signal into its elementary frequency components. After applying DCT, most of the energy of a signal is concentrated into top left corner of an image. Due to this property, DCT is widely used in image compression. This property also helps in watermarking for selecting appropriate frequency coefficients to embed the watermark.

\section{Singular Value Decomposition (SVD)}

Singular Value Decomposition is a matrix factorization technique having many applications in image processing. Since digital image is a two dimensional matrix, SVD can be applied to it. If $\mathrm{I}$ is a digital image of dimension $\mathrm{M}^{*} \mathrm{~N}$, then applying SVD on I decomposes it into three matrices $\mathrm{U}, \mathrm{S}$ and $\mathrm{V}$ with following relationship.

$$
\mathrm{I}=\mathrm{USV}^{\mathrm{T}}
$$

Here $\mathrm{U}$ is a $\mathrm{M} * \mathrm{M}$ unitary matrix, $\mathrm{V}$ is a $\mathrm{N}^{*} \mathrm{~N}$ unitary matrix and $S$ is $M * N$ matrix whose first $r$ diagonal values are Eigen values of positive definite matrix $\mathrm{I}^{\mathrm{T}} * \mathrm{I}$. Coefficients of matrix $\mathrm{U}, \mathrm{S}$ or $\mathrm{V}$ can be appropriately selected and altered for watermark embedding.

\section{PROPOSED METHOD}

In this paper a hybrid approach for watermark embedding and extraction has been proposed. Two combinations have been used to compare their performances. First combination is of DWT, DCT and SVD, whereas second combination is of DWT, Walsh and SVD. Thus main aim here is to compare performance of DCT and Walsh when combined with DWT and SVD. Further different frequency sub-bands (HL2, HH2 and $\mathrm{HH} 1$ ) of host image are tried for embedding purpose in order to observe the effect of frequency band selection on robustness and perceptibility. Experiments are carried out on 10 different color host images of size $256 * 256 * 8$ by embedding five different color images / logos of size $128 * 128 * 8$ into each host image. Let $\mathrm{H}$ be the host image and $\mathrm{W}$ be the watermark. WI refers to watermarked image. Embedding and Extraction algorithms given below are for HL2 Frequency sub-band. Same steps are conducted for HH2 and $\mathrm{HH} 1$ sub-band. For using $\mathrm{HH} 1$ frequency sub-band to embed watermark single level discrete wavelet transform is taken instead of two level DWT.

\section{A. Embedding Algorithm}

Embedding algorithm further can be subdivided into four sub-processes: a) Transformation of host image, b) Transformation of watermark, c) Embedding process and d) Generating stego image. Each of these are explained below.

\section{a) Transformation of host image}

1) Apply two level Discrete wavelet transform on host image $H$ separately on each plane. This gives us the wavelet transformed image $H$ ' of size $64 * 64 * 8$. We also get an image which can be distinguished into four different frequency bands namely LL2, HL2, LH2 and HH2.

2) On HL2 sub-band of individual plane of wavelet transformed image i.e. H', apply DCT/WALSH transform. This results into DCT/WALSH transformed image say $H^{\prime \prime}$.

3) Arrange $H$ " in zigzag manner and then form four quadrants out of it say Q1, Q2, Q3and Q4 of size $32 * 32 * 8$ each.

\section{b) Transformation of watermark}

4) Repeat step 1 and step 2 on watermark image $W$ to get $W$ " of size $32 * 32 * 8$.

5) Apply Singular Value Decomposition on each quadrant obtained in step 3. This decomposes each quadrant into 3 matrices $U, S$ and $V . S$ is the singular value matrix used for embedding purpose. 
6) Apply Singular Value Decomposition on $W$ " obtained in step 4. This decomposes $W$ " into 3 matrices $U$ ', $S$ ' and $V$ '.

c) Embedding watermark

7) Scale the $S$ matrix of each quadrant of $H$ " by value say K using Equation (1) to get S". Different values of scaling factor $k$ have been tried out to observe its effect on robustness and perceptibility.

$$
\mathrm{S}^{\prime \prime}=\mathrm{S}+\mathrm{KS}^{\prime}
$$

d) Generating stego image

8) Using $S^{\prime \prime}$, reconstruct quadrants of $H^{\prime \prime}$. i.e. $Q i{ }^{\prime}=$ $U * S * * V$.

9) Rearrange these new quadrants by inversing the zigzag procedure to get modified H".

10) Take inverse DCT/WALSH of modified H" to get H'.

11) Take two- level inverse Discrete Wavelet Transform of H' obtained in Step 10 to get watermarked image WI.

\section{B. Extraction Algorithm}

Similar to embedding algorithm, extraction algorithm is divided into three sub-processes: a) Transformation of watermarked image, b) Extraction of watermark, c) Reconstruction of watermark. Each of these are explained below.

a) Transformation of host image

1) Apply two-level Discrete wavelet transform on watermarked image WI separately on each plane. This gives us the wavelet transformed image WI' of size $64 * 64 * 8$. This image can be distinguished into four different frequency bands namely $L L 2, H L 2, L H 2$ and $H H 2$.

2) On HL2 sub-band of individual plane of wavelet transformed image i.e. WI', apply DCT/WALSH transform. This results into DCT/WALSH transformed image say WI".

3) Arrange WI" in zigzag manner and then form four quadrants out of it say $Q 1, Q 2, Q 3$ and $Q 4$ of size $32 * 32 * 8$ each.

4) Apply Singular Value Decomposition on each quadrant obtained in step 3. This decomposes each quadrant into 3 matrices $U$, $S$ and $V$.

b) Extraction of watermark

5) Extract singular values from watermarked image using modified and original singular values of $R G B$ planes of host image using Equation (2).

$$
\mathrm{S}^{\prime}=\left(\mathrm{S}^{\prime \prime}-\mathrm{S}\right) / \mathrm{K}
$$

c) Reconstruction of watermark

6) These extracted singular values are then used to construct DCT/Walsh transform coefficients of watermark say $W$ " from each quadrant.

7) Take inverse DCT/Walsh transform of W" to get W'.

8) Take inverse wavelet transform of 'W' to get extracted watermark $E W$.

Table I below shows host images of size $256 * 256 * 8$ used for experimentation. Images from left to right and top to bottom are Lena, Mandrill, Peppers, Balls, Puppy, Tiger, Flower, Ganesh, Titanic and Waterlili.

TABLE I. HOST IMAGES USED FoR EXPERIMENTATION

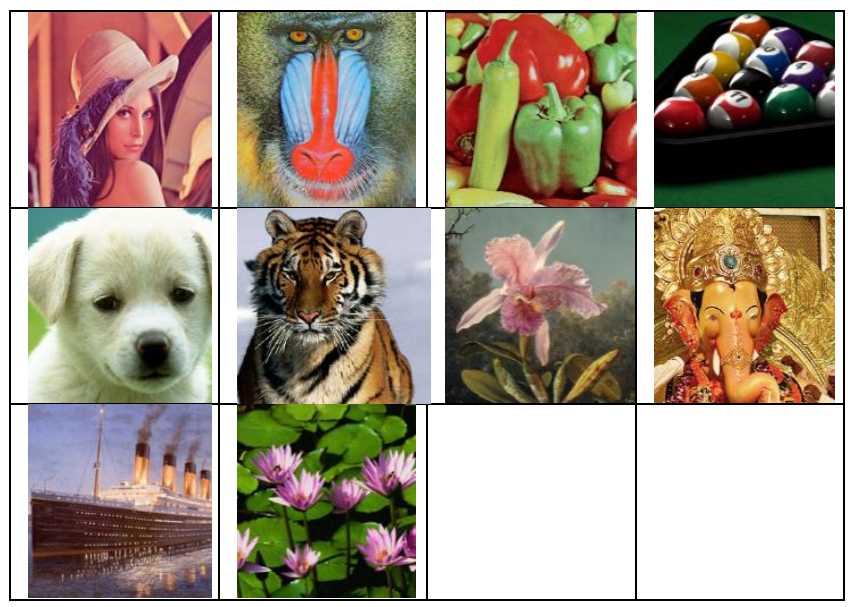

Table II below shows five different logos/images of size $128 * 128 * 8$ used as watermark. Images from left to right and top to bottom are NMIMS, Austral, Bear, Logo and CCD.

TABLE II. WATERMARK IMAGES USED FOR EXPERIMENTATION

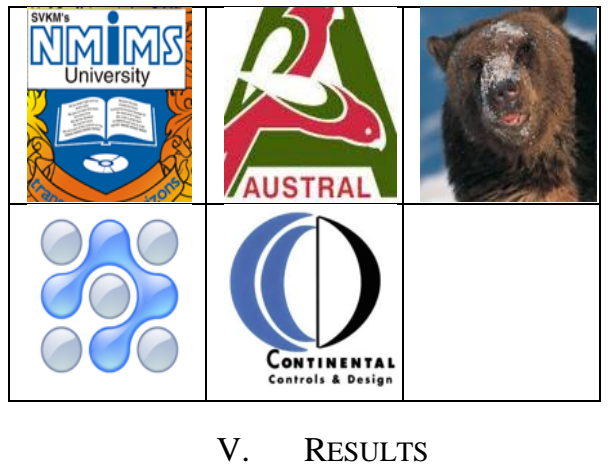

C. Results for embedding process using DCT and Walsh with $D W T-S V D$

Table III on next page shows host image Lena after embedding watermark into its HL2, HH2 and $\mathrm{HH} 1$ frequency components using DCT. These results are for $\mathrm{K}=0.05$ (except HH1), 0.1, 0.2, 0.4 and 0.6. It can be seen that, as scaling factor is increased $(0 \leq \mathrm{K} \leq 1)$, quality of host image is degraded. This is due to considerable changes taking place into singular values of frequency components of host image with increased value of $\mathrm{K}$. Table IV shows host image Lena after embedding watermark into its $\mathrm{HL} 2, \mathrm{HH} 2$ and $\mathrm{HH} 1$ frequency components using Walsh. Observations for Walsh are also similar to that of DCT.

Comparisons of results obtained for DWT-DCT-SVD and DWT-Walsh-SVD combinations are shown in following graphs. Fig. 1 shows comparison of Mean Absolute Error (MAE) between host image and watermarked image for different values of scaling factor $\mathrm{K}$, when watermark is embedded in HL2 sub-band using DCT and Walsh with DWTSVD. 


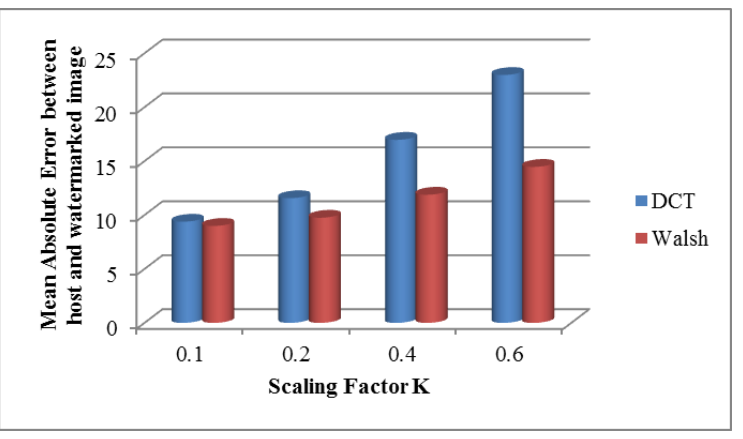

Fig. 1. Comparison of Mean Absolute Error (MAE) between host image and watermarked image for different values of scaling factor $\mathrm{K}$ when watermark is embedded in HL2 sub-band using DCT and Walsh with DWTSVD.

From Fig. 1 it can be observed that Walsh transform shows more imperceptibility than DCT for all scaling facto values. Fig. 2 and Fig. 3 show Mean Absolute Error between host and watermarked image for different scaling factor $\mathrm{K}$ when watermark is embedded in $\mathrm{HH} 2$ and $\mathrm{HH} 1$ sub-bands respectively with DWT-SVD.

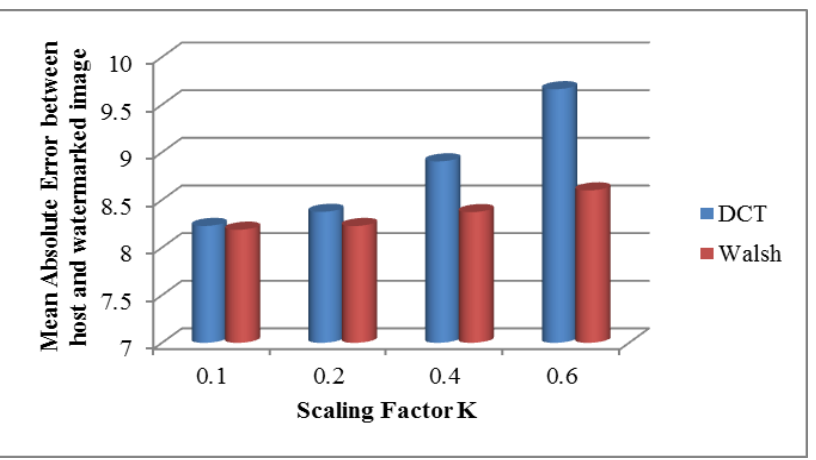

Fig. 2. Comparison of Mean Absolute Error (MAE) between host image and watermarked image for different values of scaling factor $\mathrm{K}$ when watermark is embedded in HH2 sub-band using DCT and Walsh with DWTSVD.

Fig. 2 clearly shows that Walsh transform with DWT-SVD for HH2 sub-band is more imperceptible than DCT with DWT-SVD.

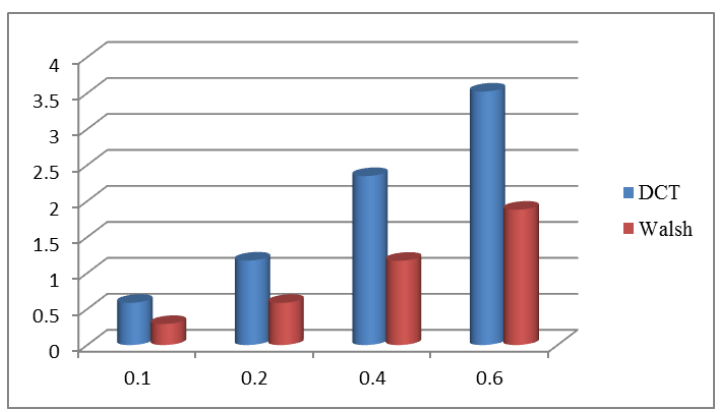

Fig. 3. Comparison of Mean Absolute Error (MAE) between host image and watermarked image for different values of scaling factor $\mathrm{K}$ when watermark is embedded in HH1 sub-band using DCT and Walsh with DWTSVD.

Difference in imperceptibility for DCT and Walsh is more significant for HH1 sub-band as shown in Fig. 3.
To summarize, from Fig. 1, Fig. 2, and Fig. 3, it can be observed that distortion caused in host image due to embedding watermark is much less for Walsh as compared to DCT in all three frequency sub- bands, i.e. Walsh shows higher imperceptibility than DCT. It also indicates that, embedding watermark into high frequency components leads to higher imperceptibility which is a requirement for good watermarking technique. Though higher frequency components are more susceptible to various image processing attacks especially image compression, it is affordable in watermarking. The reason is that, main purpose of watermarking is to provide authentication of data contents which makes the image compression issue secondary. MAE can be directly related to perceptibility because it is the absolute difference between two images and hence noticeable by Human Visual System (HVS). Table VI shows result images for watermark extraction when no attacks are performed on watermarked image $(\mathrm{K}=0.6)$ for $\mathrm{HL} 2, \mathrm{HH} 2$, HH1 sub-band using DCT. 
TABLE III.

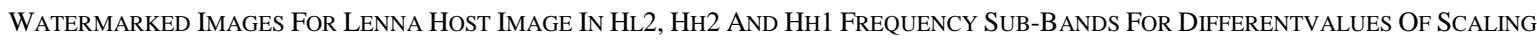
FACTOR K USING DWT-DCT-SVD

\begin{tabular}{|c|c|c|c|}
\hline \multirow{2}{*}{$\begin{array}{r}\text { Scal } \\
\text { ing Factor }(\mathbf{K}) \\
\end{array}$} & \multicolumn{3}{|c|}{ Watermarked Images (DWT-DCT-SVD) } \\
\hline & $H L 2$ & $\mathrm{HH} 2$ & HHI \\
\hline $\mathrm{K}=0.05$ & & & - \\
\hline RMSE & 9.8622 & 9.5669 & - \\
\hline MAE & 5.6053 & 5.0714 & - \\
\hline \multicolumn{4}{|l|}{$\mathrm{K}=0.1$} \\
\hline RMSE & 10.754 & 9.6301 & 0.97263 \\
\hline MAE & 6.7203 & 5.1099 & 0.56891 \\
\hline \multicolumn{4}{|l|}{$\mathrm{K}=0.2$} \\
\hline RMSE & 13.755 & 9.8784 & 1.9453 \\
\hline MAE & 9.6149 & 5.2676 & 1.1378 \\
\hline \multicolumn{4}{|l|}{$\mathrm{K}=0.4$} \\
\hline RMSE & 21.985 & 10.814 & 3.8905 \\
\hline MAE & 16.365 & 5.8456 & 2.2756 \\
\hline \multicolumn{4}{|l|}{$\mathrm{K}=0.6$} \\
\hline RMSE & 31.2 & 12.214 & 5.8358 \\
\hline MAE & 23.544 & 6.6577 & 3.4134 \\
\hline
\end{tabular}

TABLE IV.

WATERMARKED IMAGES FOR LENNA HOST IMAGE IN HL2, HH2 AND HH1 FREQUENCY SUB-BANDS FOR DIFFERENTVALUES OF SCALING FACTOR K USING DWT-WALSH-SVD

\begin{tabular}{|c|c|c|c|}
\hline \multirow{2}{*}{$\begin{array}{r}\text { Scal } \\
\text { ing Factor }(\mathbf{K}) \\
\end{array}$} & \multicolumn{3}{|c|}{ Watermarked Images (DWT-WALSH-SVD) } \\
\hline & $H L 2$ & $\mathrm{HH} 2$ & HHI \\
\hline $\mathrm{K}=0.05$ & & & - \\
\hline RMSE & 9.623 & 9.551 & - \\
\hline MAE & 5.235 & 5.062 & - \\
\hline
\end{tabular}




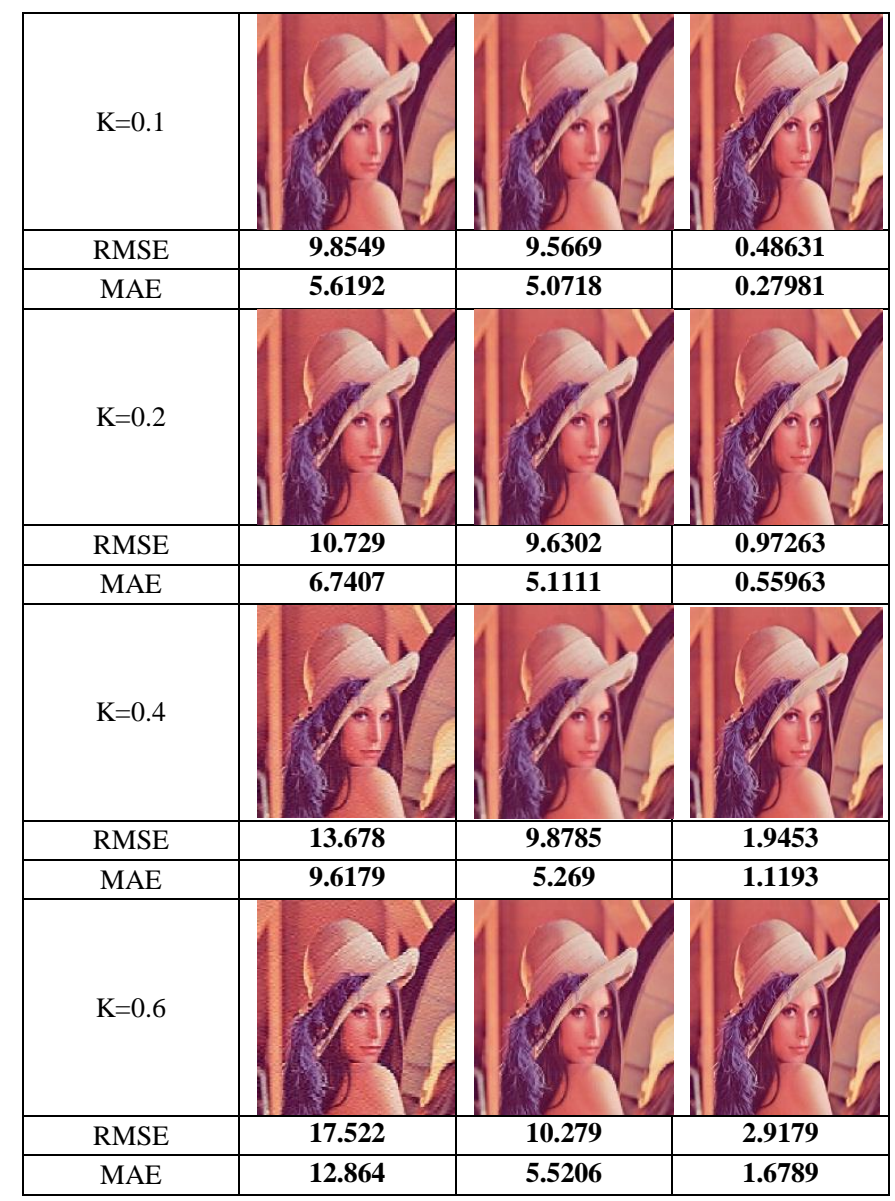

\section{Attacks on watermarked images:}

Generally attacks on digital image can be categorized into two groups. Attacks which affect the pixel values of image and which affect geometry of image [7]. In the work presented in this paper, five different types of attacks have been performed. These attacks are contrast stretching, image cropping, Gaussian noise, histogram equalization and image resizing. Table $\mathrm{V}$ below shows Lena image watermarked with 'NMIMS' image ( $\mathrm{K}=0.6)$ after performing various attacks on it. Images in Fig. 4 from left to right and top to bottom correspond to contrast stretching, cropping, adding Gaussian noise (0.1 variance), histogram equalization, and resizing. Robustness plays an important role here because, watermark should survive the attacks performed on host image for successful authentication. Due to space constraints, results of watermark extraction without any attack are shown in Table VI only for $\mathrm{K}=0.6$ for HL2, HH2 and HH1 sub-band with DWT-DCT-SVD. Table VII shows results of watermark extraction without any attack for $\mathrm{K}=0.6$ for $\mathrm{HL} 2, \mathrm{HH} 2$ and HH1 sub-band with DWT-Walsh-SVD.
TABLE V. VARIOUS ATTACKS ON LENA IMAGE AFTER EMBEDDING 'NMIMS' IMAGE INTO IT (A) CONTRAST STRETCHING (B) CROPPING (C) GAUSSIAN NOISE (D) HISTOGRAM EQUALIZATION (E) RESIZING

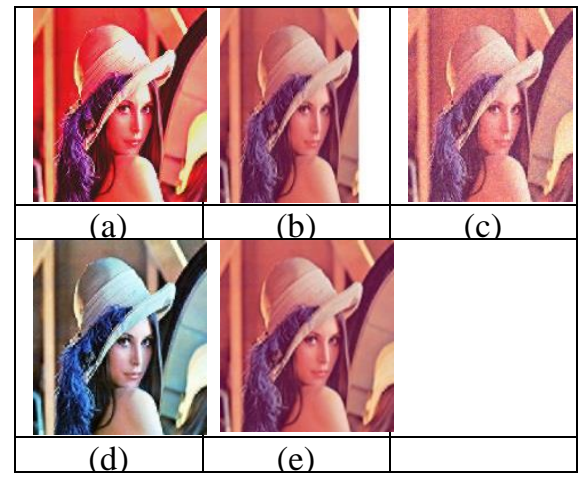

E. Results of watermark extraction from $\mathrm{HL} 2, \mathrm{HH} 2$ and HH1 sub-bands against various attacks using DCT with DWT-SVD:

Fig. 4(a), (b), (c) and (d) below show performance comparison of different sub-bands against various attacks for $\mathrm{K}=0.1,0.2,0.4,0.6$ using DCT. 


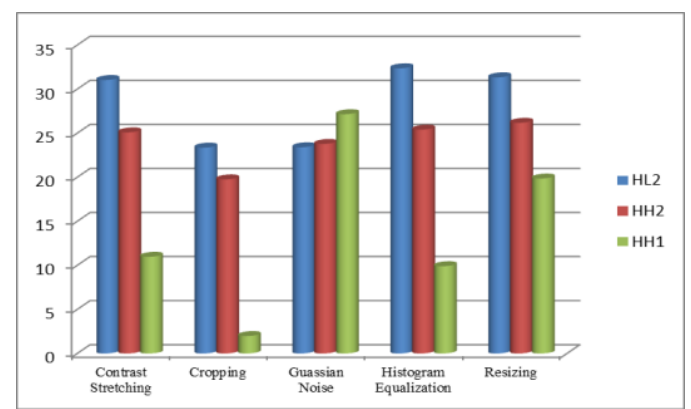

Fig. 4. .(a) Mean Absolute Error between original and extracted NMIMS watermark from $\mathrm{HL} 2, \mathrm{HH} 2$ and $\mathrm{HH} 1$ for $\mathrm{K}=0.1$ and $\mathrm{DCT}$

From Fig.4 (a), it can be noticed that for different attacks performed on watermarked image with $\mathrm{K}=0.1$, $\mathrm{HH} 1$ subband gives smaller value of MAE than HL2 and HH2 subbands (except for Gaussian noise attak.). This in turn indicates more robustness when watermark is embedded in HH1 sub-band.

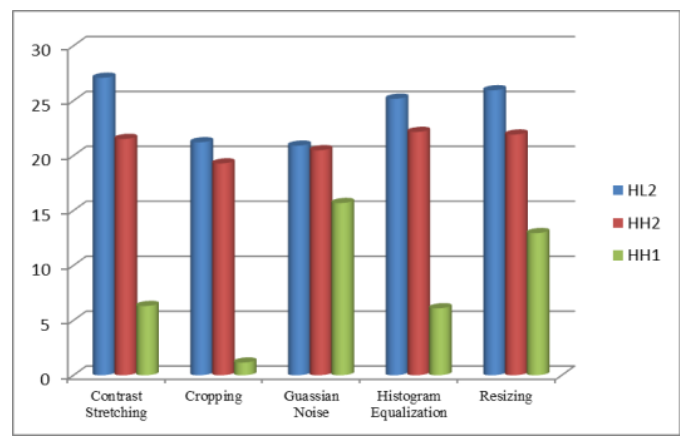

Fig. 4. (b) Mean Absolute Error between original and extracted NMIMS watermark from HL2, HH2 and HH1 for $\mathrm{K}=0.2$ and DCT.

However, from Fig. 4(b) it is observed that with $\mathrm{K}=0.2$, for all attacks, HH1 gives smallest value of MAE. Also these MAE values are smaller as compared to MAE values for $\mathrm{K}=0.1$ in previous case.

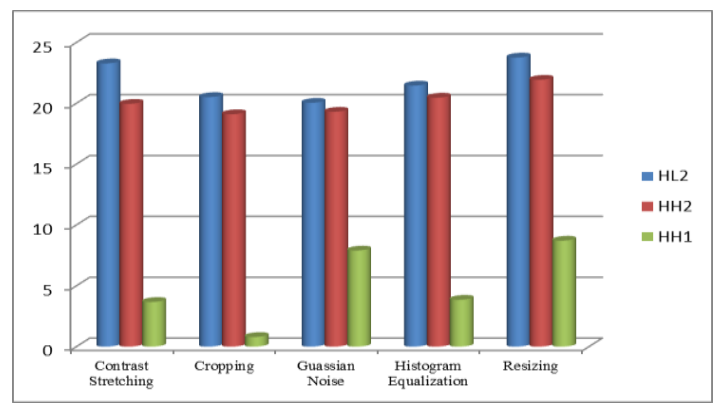

Fig. 4. (c) Mean Absolute Error between original and extracted NMIMS watermark from HL2, HH2 and $\mathrm{HH} 1$ for $\mathrm{K}=0.4$ and DCT.

From Fig. 4.(c), we can say that HH1 is much better in robustness than $\mathrm{HL} 2$ and $\mathrm{HH} 2$ for $\mathrm{K}=0.4$. MAE values are further reduced with increase in value of $\mathrm{K}$.

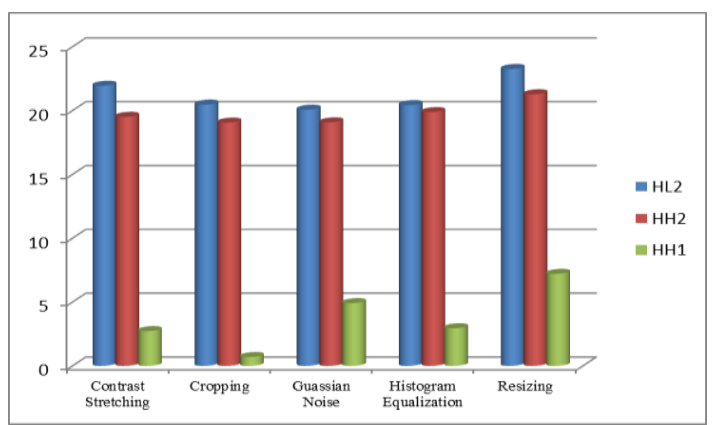

Fig. 4. (d) Mean Absolute Error between original and extracted NMIMS watermark from HL2, HH2 and $\mathrm{HH} 1$ for $\mathrm{K}=0.6$ and DCT

This improvement in robustness for $\mathrm{HH} 1$ sub-bands continues for higher value of $\mathrm{K}(\mathrm{K}=0.6)$ as shown in Fig.4 (d),

Thus it can be concluded that as we increase the value of scaling factor, watermark recovered from attack are closest to original watermark for $\mathrm{HH} 1$ sub-band. Similar results are observed for Walsh with DWT-SVD.

Further, for each sub-band, robustness of DCT and Walsh is compared by considering average MAE between original and extracted watermark for different attacks and for different values of $\mathrm{K}$. For this, average MAE is computed over 10 host images for each attack in HL2, HH2 and HH1 sub-band separately. It is observed that robustness shown by Walsh transform is acceptable with less computational cost for each sub-band except for Gaussian noise in HH1 sub-band. This Comparison of robustness (MAE) for DCT and Walsh with $\mathrm{K}=0.6$ is shown in Fig. 5(a)-(c). Watermarks extracted from each quadrant of $\mathrm{HH} 1$ sub-band for various attacks and $\mathrm{K}=0.6$ using DWT-DCTSVD and DWT-Walsh-SVD are shown in Table VIII and Table IX respectively.

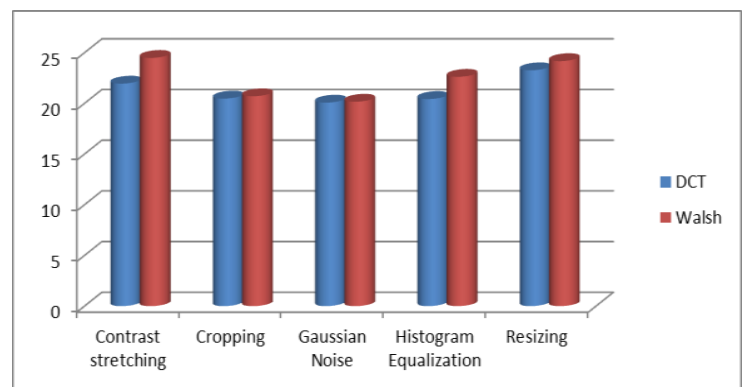

Fig. 5. a) Comparison of average MAE for DCT and Walsh for HL2 sub-band ( $\mathrm{K}=0.6$, 'NMIMS' Watermark)

From Fig. 5(a), it is observed that MAE between original and extracted watermark from HL2 sub-band is slightly more for Walsh as compared to DCT and hence it is acceptable. 


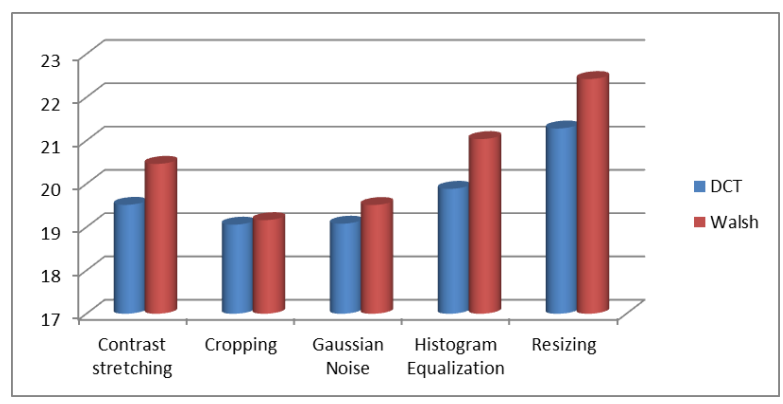

Fig. 5. (b) Comparison of average MAE for DCT and Walsh for $\mathrm{HH} 2$ sub-band ( $\mathrm{K}=0.6$, 'NMIMS' Watermark)

From Fig. 5(b), it is observed that MAE between original and extracted watermark from $\mathrm{HH} 2$ sub-band is slightly increased for Walsh. It is still acceptable because
MAE for embedding process using Walsh is better than

DCT.

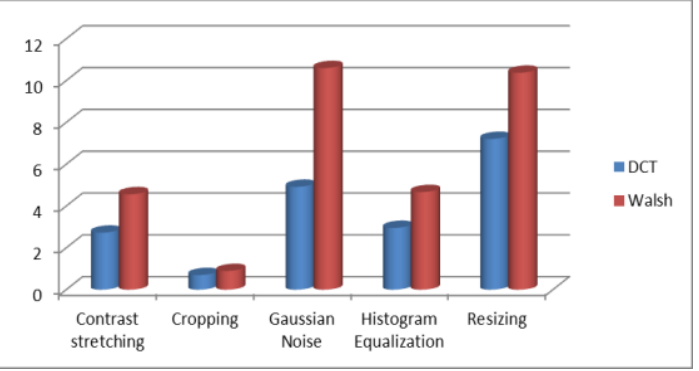

Fig. 5. (c) Comparison of average MAE for DCT and Walsh for HH1 sub-band ( $\mathrm{K}=0.6$, 'NMIMS' Watermark)

From Fig.5(c), it can be said that the performance of Walsh is acceptable for extraction from $\mathrm{HH} 1$ sub-band since MAE values are much smaller as compared to MAE values for $\mathrm{HL} 2$ and $\mathrm{HH} 2$ sub-band for watermark extraction process

TABLE VI. WATERMARKE EXTRACTED FROM FOUR QUADRANTS OF HL2, HH2 AND HH1 SUB-BAND OF LENA HOST IMAGE FOR K =0.6USING DWTDCT-SVD WHEN NO ATTACK IS PERFORMED ON IT.

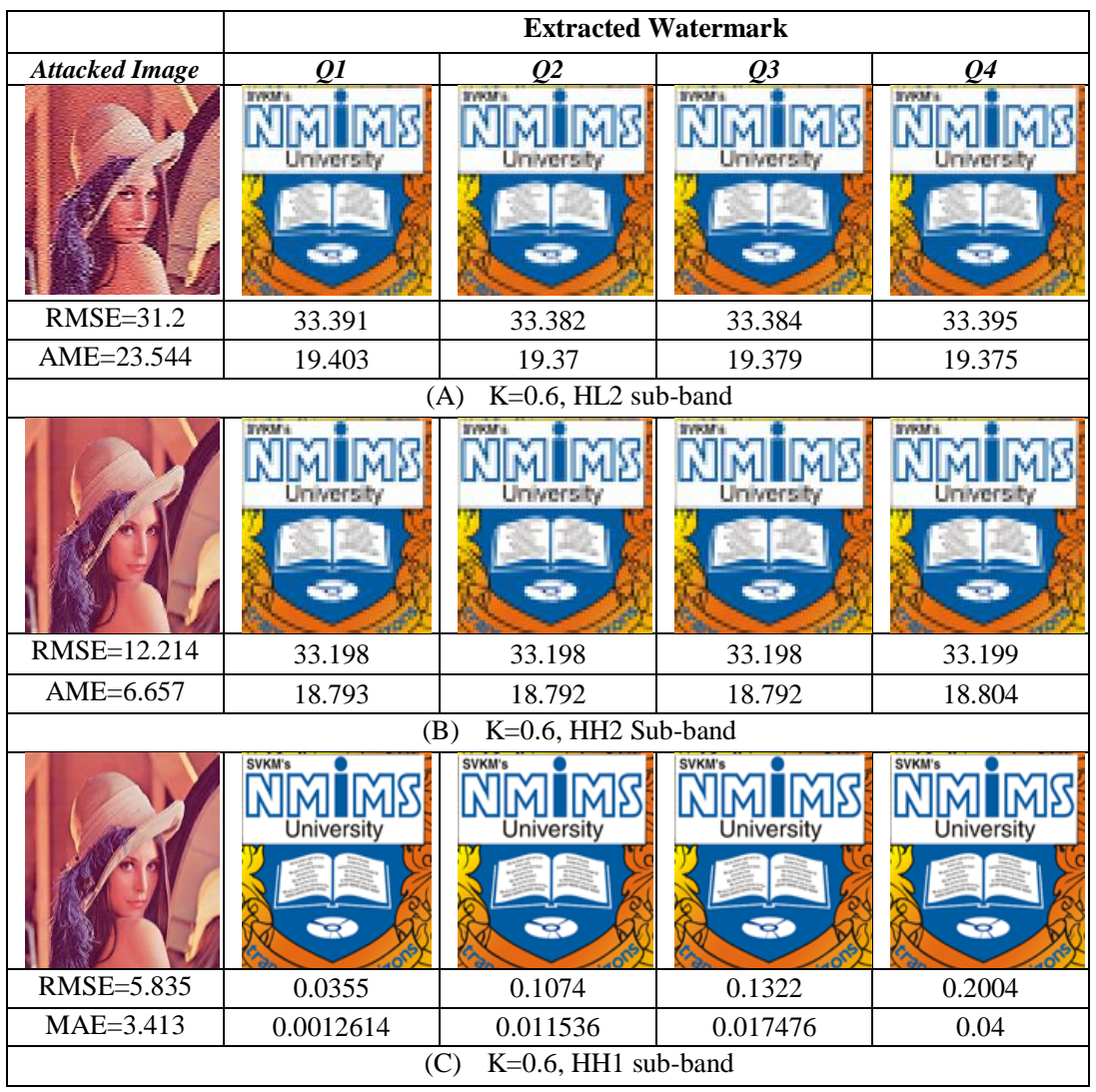

TABLE VII.

WATERMARKE EXTRACTED FROM FOUR QUADRANTS OF HL2, HH2,HH1 SUB-BAND OF LENA HOST IMAGE FOR K=0.6 USING DWTWALSH-SVD WHEN NO ATTACK IS PERFORMED ON IT.

\begin{tabular}{|l|c|c|c|c|}
\hline & \multicolumn{4}{|c|}{ Extracted Watermark } \\
\hline Attacked Image & $Q 1$ & $Q 2$ & $Q 3$ & $Q 4$ \\
\hline
\end{tabular}




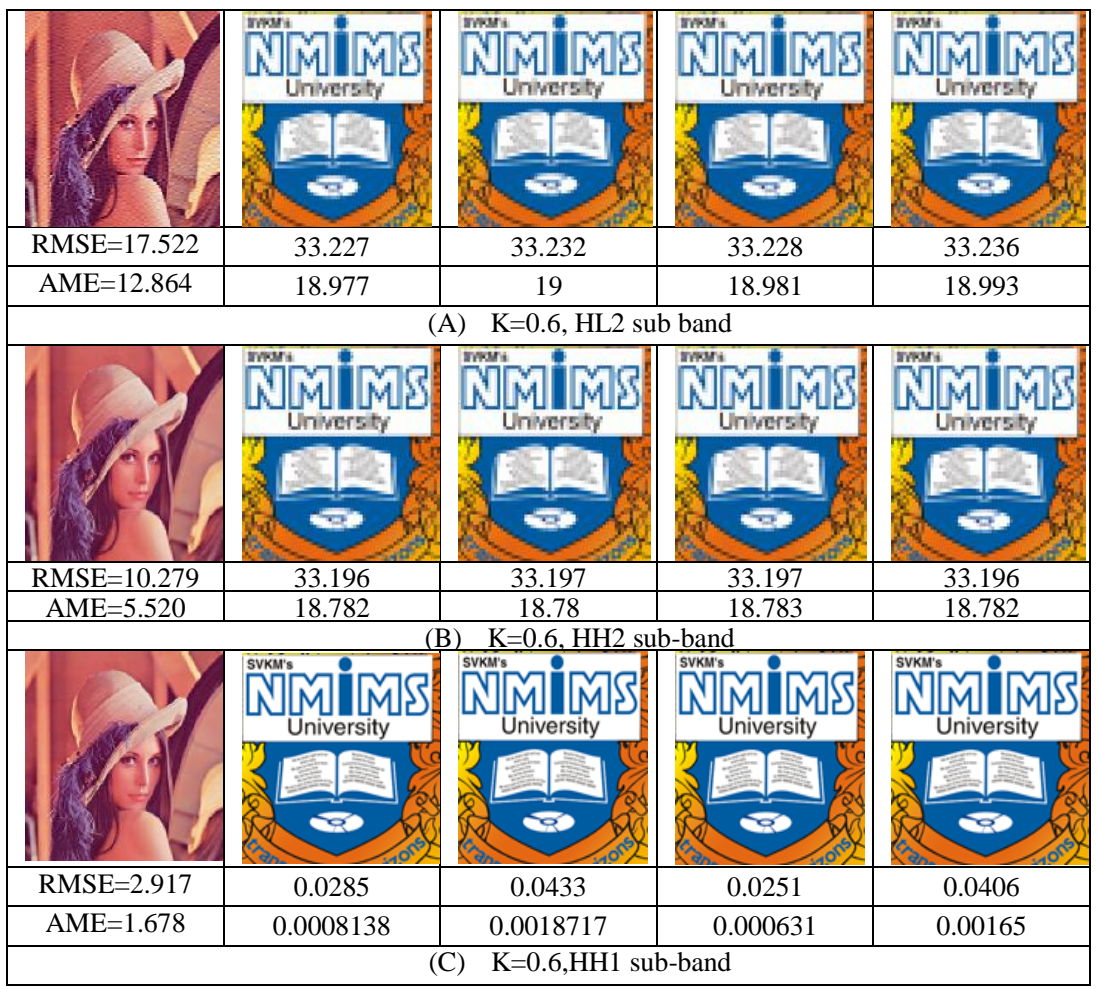

TABLE VIII. WATERMARKE EXTRACTED FROM FOUR QUADRANTS OF HH1 SUB-BAND OF LENA HOST IMAGE FOR K=0.6 USING DWT-DCT-SVD FOR (A) CONTRAST STRETCHING, (B) CROPPING, (C) GAUSSIAN NOISE, (D) HISTOGRAM EQUALIZATION (E) IMAGE RESIZING ATTACKS.

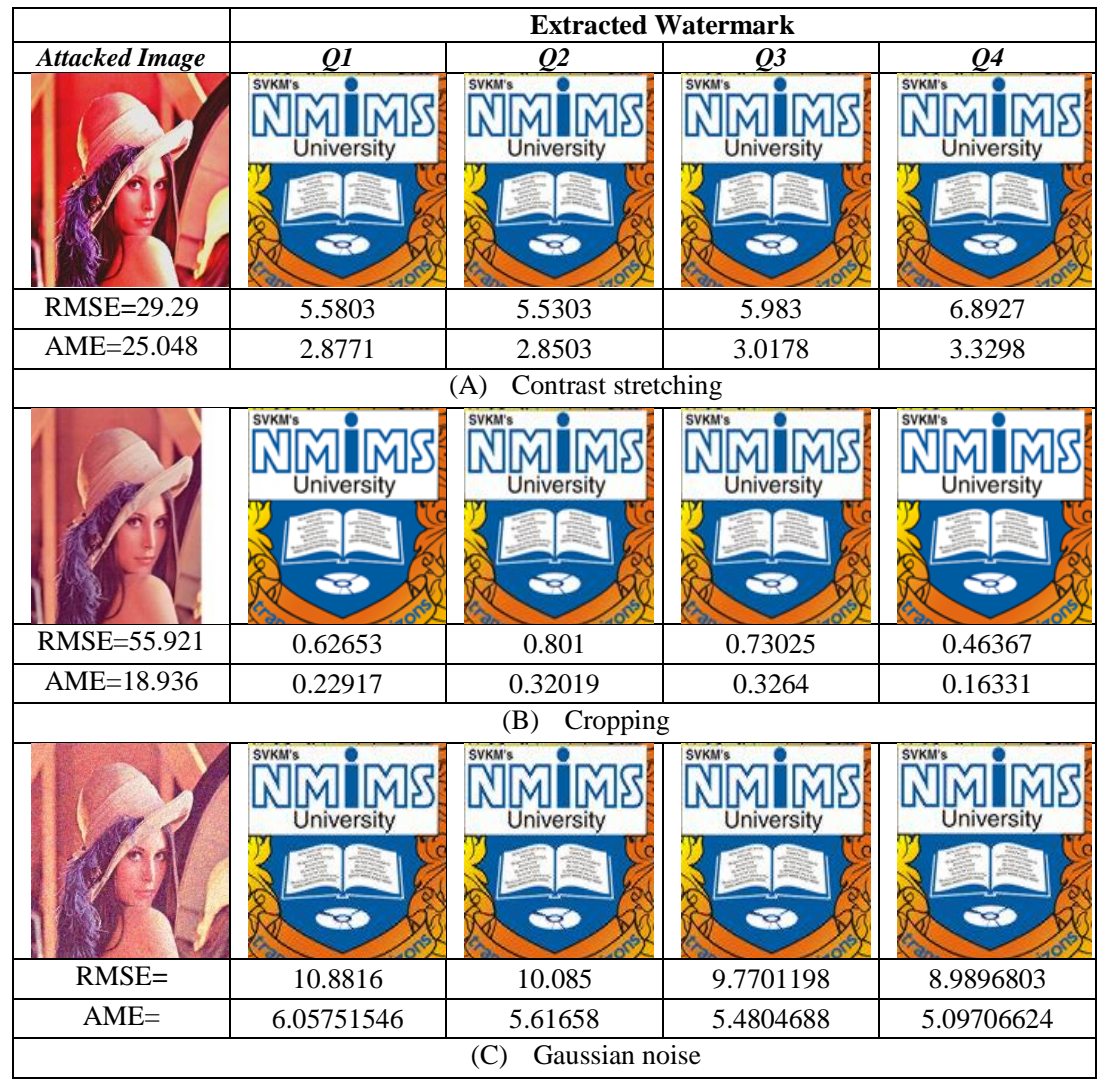




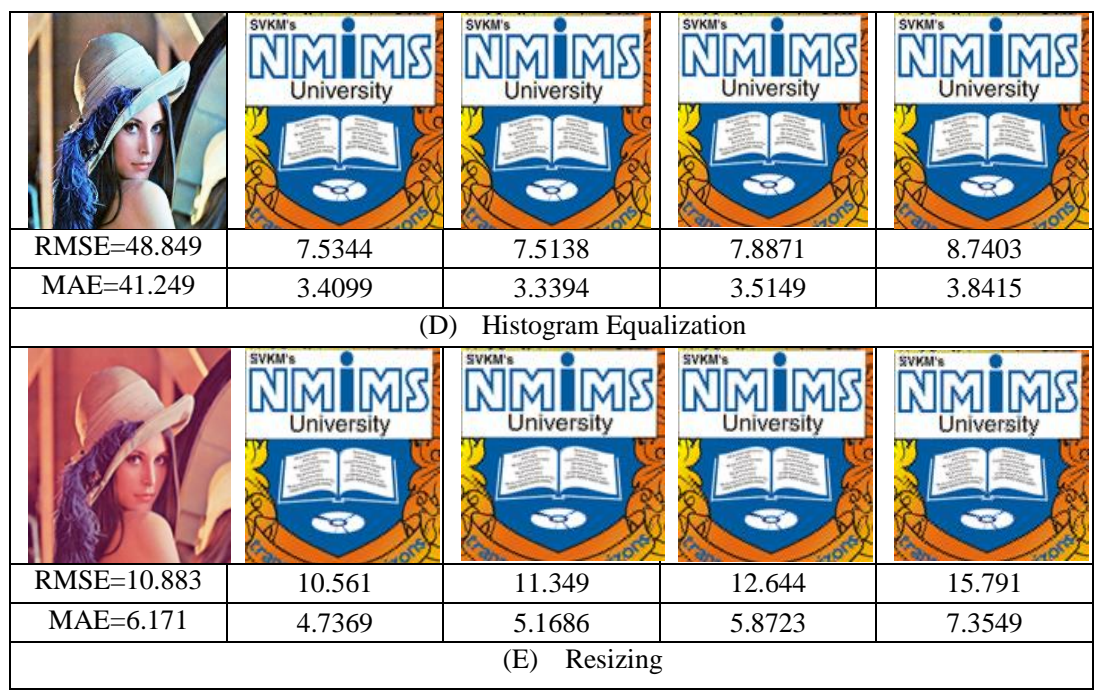

TABLE IX. WATERMARKE EXTRACTED FROM FOUR QUADRANTS OF HH1 SUB-BAND OF LENA HOST IMAGE FOR K=0.6 USING DWT-WALSH-SVD FOR (A) CONTRAST STRETCHING, (B) CROPPING, (C) GAUSSIAN NOISE, (D) HISTOGRAM EQUALIZATION (E) IMAGE RESIZING ATTACKS.

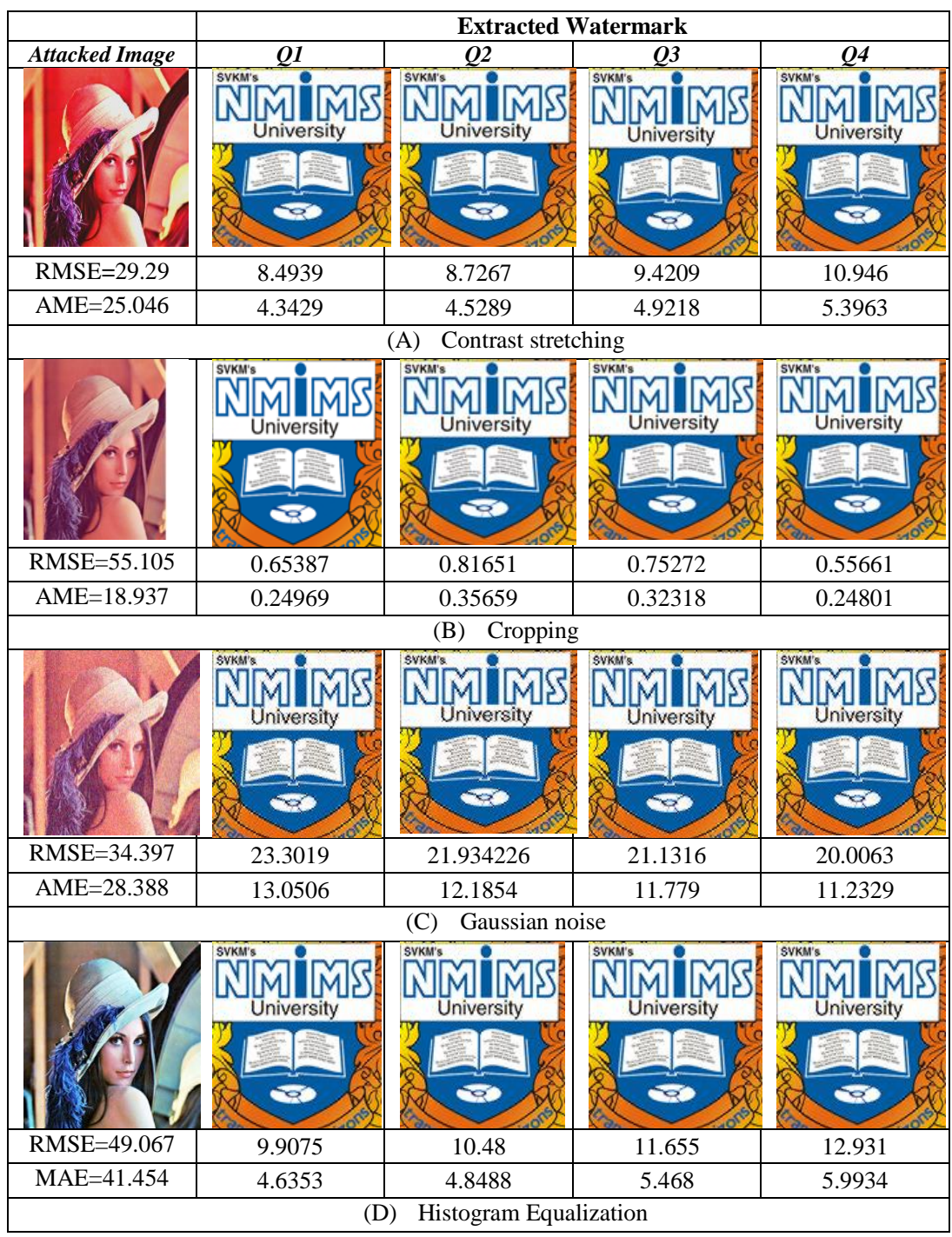




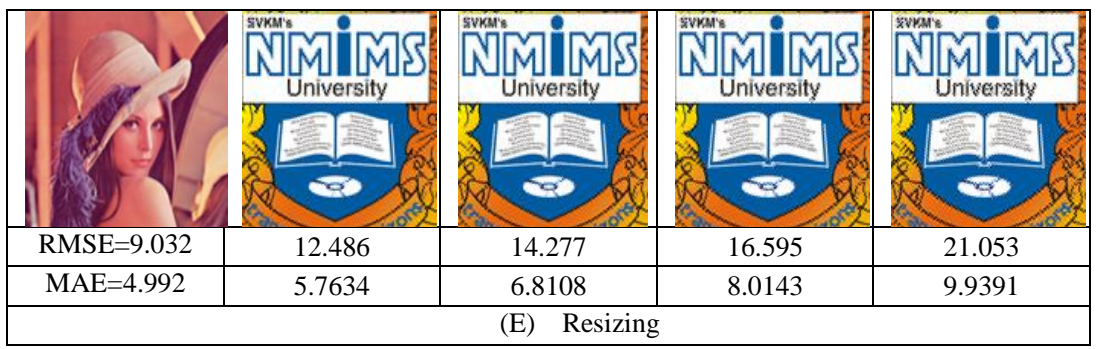

\section{CONCLUSION AND FURTHER WORK}

Following conclusions can be drawn based on the work presented in this paper. As value of scaling factor increases, MAE between host image and watermark becomes significant thereby reducing imperceptibility. However, loss of perceptibility is less when watermark is embedded in high frequency components of host image. Since high frequency components of an image correspond to edges and borders of an image, embedding watermark causes distortion in images. But this distortion is affordable as compared to distortion in image caused by embedding watermark in HL or LH frequency components. Frequently, in image processing attacks, high frequency components are eliminated which results into loss of watermark information. However, such elimination is possible or can be of major concern in data compression. In watermarking, main emphasis is on protecting copyright information or content identification and not on data compression. Thus, it is acceptable to embed the watermark image in high frequency components rather than in low or medium frequency components. Walsh transform when used with DWT-SVD results in computationally faster watermarking scheme. Robustness and imperceptibility provided by Walsh is acceptable when compared with DWTDCT-SVD.

Further work includes use of different orthogonal transforms like slant, Hartley, Kekre's transform and wavelet transforms obtained from them for watermarking.

\section{REFERENCES}

[1] Smitha Rao, Jyothsna A. N, Pinaka Pani. R, "Digital watermarking: applications,techniques and attacks", International Journal of Computer Applications Volume 44, No. 7, pp. 29-34, April 2012.

[2] Chih-chin lai, Cheng-chih Tsai, "Digital image watermarking using discrete wavelet transform and singular value decomposition", IEEE Transaction on Instrumentation and Measurement, Vol. 59, No. 11, pp. 3060-3063.

[3] Basia Gunjal, R. Manthalkar,"An overview of transform domain robust digital image watermarking algorithms", Journal of Emerging Trends in Computing and Information Science, Vol. 2, No. 1, 2010-11, pp.37-42.

[4] Harry Andrews,"Singular value decompositions and digital image processing", IEEE Transactions on Acoustic, Speech and Signal Processing, Vol. 24, No. 1, 1976, pp. 26-53.

[5] Xi-Ping and Qing-Sheng Zhu, "A robust wavelet-domain watermarking algorithm for color image", Proceedings of the Fifth International Conference on Machine Learning and Cybernetics, Dalian, pp.13-16 August 2006

[6] A Mansouri, A Mahmoudi Aznaveh, F Azar, "SVD-based digital image watermarking using complex wavelet transform", Sadhana, Vol. 34, Part 3, pp. 393-406, June 2009.

[7] Rashmi Agarwal, K. Venugopalan, "Digital watermarking of color images in the singular domain", IJCA Special issue on "Computational Science- New Dimensions \& Perspectives, pp. 144-149, 2011.
[8] S. Murty, Dr. Rajesh Kumar, "A Robust Digital Image Watermarking Scheme using Hybrid DWT-DCT-SVD Technique", IJCSNS, Vol.10,No.10, pp. 185-192, Oct 2010.

[9] Satendra Kumar, Ashwini Saini, Papendra Kumar, "SVD based Robust Digital Image Watermarking using Discrete Wavelet Transform”, IJCA, Vol. 45No. 10, pp.7-11, May 2012.

[10] R. Krishnamoorthi, Sheba Kezia,'Image Adaptive Watermarking with Visual Model in Orthogonal Polynomials based Transformation Domain",IJICE, 5:2, pp. 146-153, 2009.

[11] Ko-Ming Chan, Long-wen Chang, "A Novel Public Watermarking System based on Advanced Encryption System", IEEE Proc.of $18^{\text {th }}$ International Conference on Advanced Information Networking and Application, 2004.

[12] Veysel Aslantas, A. Latif Dogan and Serkan Ozturk, "DWT-SVD based image watermarking using particle swarm optimizer", Proc. Of IEEE International Conference on Multimedia and Expo, 2008 pp. 241-244.

[13] Yang Quianli, Cai Yanhong,"A digital watermarking algorithm based on DWT and DCT",IEEE International Symposium on Information Technology in Medicine and Education, 2012, pp. 1102-1105.

\section{AUTHORS PROFILE}

Dr. H. B. Kekre has received B.E. (Hons.) in Telecomm. Engg. from Jabalpur University in 1958, M.Tech (Industrial Electronics) from IIT Bombay in 1960, M.S.Engg. (Electrical Engg.) from University of Ottawa in 1965 and Ph.D. (System Identification) from IIT Bombay in 1970. He has worked Over 35 years as Faculty of Electrical Engineering and then HOD Computer Science and Engg. at IIT Bombay. After serving IIT for 35 years, he retired in 1995. After retirement from IIT, for 13 years he was working as a professor and head in the department of computer engineering and Vice principal at Thadomal Shahani Engg. College, Mumbai. Now he is senior professor at MPSTME, SVKM's NMIMS University. He has guided 17 Ph.Ds., more than 100 M.E./M.Tech and several B.E. / B.Tech projects, while in IIT and TSEC. His areas of interest are Digital Signal processing, Image Processing and Computer Networking. He has more than 450 papers in National / International Journals and Conferences to his credit. He was Senior Member of IEEE. Presently He is Fellow of IETE, Life Member of ISTE and Senior Member of International Association of Computer Science and Information Technology (IACSIT). Recently fifteen students working under his guidance have received best paper awards. Currently eight research scholars working under his guidance have been awarded Ph. D. by NMIMS (Deemed to be University). At present seven research scholars are pursuing Ph.D. program under his guidance.

Dr. Tanuja K. Sarode has received M.E. (Computer Engineering) degree from Mumbai University in 2004, Ph.D. from Mukesh Patel School of Technology, Management and Engg. SVKM's NMIMS University, Vile-Parle (W), Mumbai, INDIA. She has more than 11 years of experience in teaching. Currently working as Assistant Professor in Dept. of Computer Engineering at Thadomal Shahani Engineering College, Mumbai. She is member of International Association of Engineers (IAENG) and International Association of Computer Science and Information Technology (IACSIT). Her areas of interest are Image Processing, Signal Processing and Computer Graphics. She has 137 papers in National/International Conferences/journal to her credit.

Ms. Shachi Natu has received M.E. (Computer Engineering) degree from Mumbai University in 2010. Currently pursuing Ph.D. from NMIMS University. She has 08 years of experience in teaching. Currently working as Assistant Professor in Department of Information Technology at Thadomal Shahani Engineering College, Mumbai. Her areas of interest are Image Processing, Database Management Systems and Operating Systems. She has 12 papers in International Conferences/journal to her credit. 